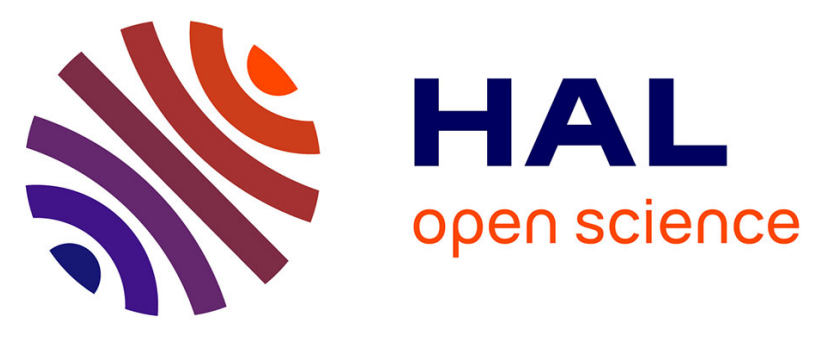

\title{
Is there a timing for sensitivity to acute cerebral ischemia in migraine patients?
}

Isabelle Francillard, Lou Grangeon, Agathe Cornillot, Estelle Houivet, Ozlem

Ozkul-Wermester, Aude Triquenot-Bagan, Benjamin Hebant, David Maltete, Emmanuel Gerardin, Evelyne Guegan-Massardier

\section{To cite this version:}

Isabelle Francillard, Lou Grangeon, Agathe Cornillot, Estelle Houivet, Ozlem Ozkul-Wermester, et al.. Is there a timing for sensitivity to acute cerebral ischemia in migraine patients?. Journal of the Neurological Sciences, 2020, 408, pp.116528 -. 10.1016/j.jns.2019.116528 . hal-03488472

\section{HAL Id: hal-03488472 \\ https://hal.science/hal-03488472}

Submitted on 21 Dec 2021

HAL is a multi-disciplinary open access archive for the deposit and dissemination of scientific research documents, whether they are published or not. The documents may come from teaching and research institutions in France or abroad, or from public or private research centers.
L'archive ouverte pluridisciplinaire HAL, est destinée au dépôt et à la diffusion de documents scientifiques de niveau recherche, publiés ou non, émanant des établissements d'enseignement et de recherche français ou étrangers, des laboratoires publics ou privés.

\section{(ㅇ)(1) $\$$}

Distributed under a Creative Commons Attribution - NonCommercial| 4.0 International 


\section{IS THERE A TIMING FOR SENSITIVITY TO ACUTE CEREBRAL ISCHEMIA IN MIGRAINE PATIENTS?}

Isabelle Francillard ${ }^{1 \#}$, Lou Grangeon ${ }^{1 \#}$, Agathe Cornillot ${ }^{2}$, Estelle Houivet ${ }^{3}$, Ozlem Ozkul,Wermester ${ }^{1}$, Aude Triquenot-Bagan ${ }^{1}$, Benjamin Hebant ${ }^{1}$, David Maltete ${ }^{1}$, Emmanuel Gerardin $^{2}$, Evelyne Guegan-Massardier ${ }^{1}$

${ }^{\#}$ Co-first authors

Affiliations:

${ }^{1}$ Department of Neurology, Rouen University Hospital, 76031, Rouen, France

${ }^{2}$ Department of Radiology, Rouen University Hospital, 76031, Rouen, France

${ }^{3}$ Department of Biostatistics and Clinical Research, INSERM U 1219, Rouen University Hospital, University of Rouen, Rouen, France

Corresponding author: Lou Grangeon, M.D.

Department of Neurology

Rouen University Hospital.

76031 Rouen Cedex, France

Tel.: +(33) 2328882 94; Fax: + (33) 232888741

E-Mail: lou.grangeon@gmail.com

Running Title: Perfusion CT during acute cerebral ischemia in migraineurs

Abstract word count: 249

Total word count: 3752

References: 19

Table 1: Demographic, clinical, and radiologic characteristics according to migraine status Figure 1: Flow chart

Figure 2: Frequency histogram of mismatch values for migraine patients and control group

Disclosures: LG, IF, AC, EH, OO, AT, DM, BH, EG and EGM have no disclosures 


\section{ABSTRACT:}

Background: Migraine may be a factor of increased cerebral sensitivity to ischemia. Previous studies were conducted within 6 to 72 after stroke onset. We aimed to determine if an accelerated infarct growth exists in migraine patients within the first 4.5 hours.

Method: A retrospective case-control study was conducted where all patients admitted for acute stroke started less than 4.5 hours before and who underwent perfusion CT were assessed. The hypoperfusion and necrosis volumes on initial CT perfusion were analyzed, as well as the final infarct volume on MRI performed within 72hours after admission. A no-mismatch pattern was defined as a ratio necrosis / hypoperfusion volume> $83 \%$.

Results: 24 patients with personal history of migraine were identified, 8 of them with aura. The control cohort included 51 patients. No difference was found between groups in terms of demographics, initial severity or outcome or presumed cause of stroke. Mean time to CT scan was 125 minutes in migraine patients and 127 minutes in the control group. A no-mismatch pattern was equally found in migraine patients and controls, even after adjustment for age, sex and presence of proximal occlusion $(\mathrm{p}=0.22)$. The final infarct volume was also similar in both groups.

Conclusions: Migraine patients did not display more no-mismatch pattern than controls within the 4.5 hours of stroke onset. This deviates from previous studies and may be due to our earlier time from stroke onset to CT scan. A history of migraine may lead to malignant progression of ischemia but occurring only after several hours.

\section{KEYWORDS:}

Migraine, cerebral ischemia, CT perfusion, infarct growth, mismatch pattern, cortical spreading depression, reperfusion 


\section{INTRODUCTION}

During acute cerebral ischemia, the penumbra is shown as a rim of tissue surrounding the severely ischemic core (or infarct core) and remains viable for several hours. Reperfusion interventions aim to salvage this moderately ischemic tissue.

Recent data in literature suggest that migraine aura may be a factor of increased cerebral sensitivity to ischemia [1]. Indeed, studies based on animal models for migraine such as the FMH1 (familial hemiplegic migraine type 1) mutant mice showed an accelerated infarct growth compared to wild type [1,2]. This growth was correlated to the cortical spreading depression, which is implicated in migraine aura pathogenesis [3].

To date, few clinical studies were performed to address this issue and further supported this hypothesis. First, Mawet et al. [4] published a retrospective study based on MRI performed in patients with acute ischemic stroke within 72 hours following onset of symptoms. More recently, Pezzini et al. [5] analyzed CT perfusion in migraine patients who underwent reperfusion therapy within 6 hours after onset of symptoms. In both studies, migraine patients were more likely to have a no-mismatch pattern, defined as more than $83 \%$ of necrosis compared to the non-migraine patients (respectively $22 \%$ versus $4 \%$ [4] and $27.9 \%$ versus $11.5 \%[5])$.

These first results were consistent with an increased vulnerability to cerebral ischemia in migraine sufferers and pave the way for further studies. Given the growing nature of the necrosis, the timing of such increased sensitivity to ischemia remains unknown and can deeply affect the efficacy of reperfusion therapies in migraine patients. Indeed, thrombolysis is most likely to be effective in the penumbra than infarct core (or necrosis) and can only be used within the first 4.5 hours [6, 7]. 
Thus, we sought to determine if an accelerated infarct growth exists in migraine patients within 4.5 hours following stroke onset. 


\section{$\underline{\text { METHODS }}$}

\section{1/ Migraine patients}

This retrospective study was conducted in neurological vascular intensive care unit (NVICU) of the Rouen University Hospital between January 2014 and December 2016.

All consecutive patients with prior diagnosis of migraine admitted for symptoms suggestive of acute stroke started less than 4.5 hours before were assessed (Figure 1- Flow chart). Migraine status was recorded in the NVICU standard medical file upon initial examination according to the International Classification of Headache Disorders - third edition (ICHD-3 $\beta$ ) diagnostic criteria [8]. Only patients who underwent the in-house stroke alert protocol including multimodal CT performed upon admission and who showed perfusion abnormality in anterior circulation allowing further mismatch evaluation were included. Definitive diagnosis of acute ischemic stroke should have been confirmed on MRI performed 24 to 72 hours after admission. Consecutive patients with a known non-migraine status admitted to NVICU during the same period, using the same diagnostic assessment were used as a control population.

Exclusion criteria were age under 18 years old, other cerebral lesion diagnosed on initial CT, contrast allergy, history of severe renal failure, pregnancy and non- available imaging (MRI or CT perfusion). The study protocol was approved by the Ethics Committee of Rouen University Hospital. We would be wiling to share the de-identified Excel database, which includes all the clinical and radiological data of the participants upon reasonable request

\section{2/ Clinical assessment}

Demographic characteristics, individual vascular risk factor, time from symptom onset to admission multimodal CT, modified RANKIN score before admission and baseline National Institute of Health Stroke Scale (NIHSS) at admission and discharge were recorded. 
According to the ICHD-3 $\beta$ diagnostic criteria, patients were classified as migraine with or without aura. For each patient, we evaluated the cause of stroke, including arteriosclerosis, small-vessel disease, cardiac embolism, cervical artery dissection and other or undetermined pathogenesis.

\section{3/ Neuroimaging protocol}

All neuroimaging were performed according to the standard local stroke alert protocol. All patients admitted for symptoms suggestive of acute stroke within $6 \mathrm{~h}$ of onset received multimodal computed tomography (CT), including a non-contrast brain CT, perfusion $\mathrm{CT}$ (PCT) and CT angiography of the cervical and intracranial arteries (CTA) with a General Electric Lightspeed 64-slice scanner. A two-phase PCT was then performed after a 47-ml injection of iodinated contrast at $4 \mathrm{ml} / \mathrm{s}$. The PCT-first phase was a 60 -second cine series, covering $4 \mathrm{~cm}$ centred on the basal ganglia. In the second phase, the same slices were obtained at 30-second intervals for additional 90 seconds. CTA was performed after a 5-min delay from the aortic arch to the vertex with $70 \mathrm{ml}$ of iodinated contrast at $3.5 \mathrm{ml} / \mathrm{s}$.

Brain MRIs were performed within 72 hours after admission on either a 1.5 or 3 Tesla scanner to determine final infarct size. DWI, gradient echo T2-weighted, fluid attenuated inversion recovery and three-dimensional time-of-flight sequences were performed.

\section{4/ Imaging analysis}

All images (CT and MRI) were analysed by the same neuroradiologist (AC) blinded to the migraine status, using a GE Healthcare workstation (ADW 4.6, GE Healthcare, Waukesha, Wis., USA). 
Regarding CTA analysis, we recorded whether there was intracranial arterial occlusion. A proximal occlusion was defined as occlusion within the first 3 segments of the intracranial arterial system.

Regarding perfusion CT, all perfusion maps were processed using OLEA SPHERE ${ }^{\circledR}$ software (Olea Medical ${ }^{\circledR}$, La Ciotat, France). Cerebral blood volume (CBV), cerebral blood flow (CBF), mean-transit-time (MTT) and Time to maximum (Tmax) maps were generated for each patient using block-circulant Singular Value Decomposition (oSVD) deconvolution method [9] - the concentration-time curves of tissue were deconvoluted by the arterial input function (AIF) to obtain a residue function. To avoid partial volume effect, the AIF curve was rescaled using a venous output function (VOF). Both AIF and VOF were automatically selected. The automatic AIF selection algorithm is based on a clustering method, which classifies curves using their area under curve, their roughness, and their first moment in order to distinguish arterial signals from tissue ones. As for VOF, the algorithm is similar to the AIF one with slight adaptations to pick up vein signals instead of arteries.

CBV was derived from the area under the convoluted concentration-time curve, CBF from the height of the impulse of the residue function, MTT from the weighted average of the area under the residue function curve, and Tmax from the delay between the beginning of the acquisition and the impulse time of the residue function.

The total hypoperfusion was then defined as the area with Tmax $>6 \mathrm{~s}$ and the infarct core (necrosis) was defined by the hypoperfused area with a relative CBF $<40 \%$ of the contralateral-side mean value and Tmax > 2s. [10].

We finally calculated the penumbra volume and absolute mismatch value, defined as the ratio necrosis / hypoperfusion volume. We used a ratio $>83 \%$ to define a no-mismatch pattern as previously reported [4]. 
Based on the MRI DWI sequences, infarct volume (in millilitres) was calculated by manual delineation of the lesion on each slice multiplied by slice thickness.

\section{5/ Statistical analyses}

We performed univariate analysis to compare clinical and imaging data between the two groups. Fisher's exact test and Chi square were used for categorical data, and the MannWhitney $U$ test was used for continuous data. In order to assess the association between migraine status and the infarct growth, we used a stepwise logistic regression. All variables assumed to be relevant or potentially implying confusion bias were fed into a multivariable regression model. All statistical analyses were performed using SPSS 16.0. The threshold for statistical significance was set at $\mathrm{p} \leq 0.05$.

\section{$\underline{\text { RESULTS }}$}

We identified 24 patients, with personal history of migraine, who fulfilled the inclusion criteria (Figure1, flow chart). Among them, 8 patients were diagnosed with migraine with aura. The control cohort included 51 patients with no migraine history. The clinical and radiological characteristics of the migraine patients and control group are described in Table 1. The mean age at inclusion in migraine patients was $63+/-13.7$ years old. No difference in terms of age was found between both groups $(\mathrm{p}=0.09)$. Migraine group included more female patients $(58 \%$ vs $33 \%, \mathrm{p}=0.04)$, in keeping with the female predominance of migraine.

No difference was found in terms of initial severity (NIHSS at admission 5.5 +/- 6.9 vs $10+/-$ $7.5, \mathrm{p}=0.26$ ), outcome at discharge (NIHSS $0+/-3.6$ vs $1+/-5.3, \mathrm{p}=0.1$ ), or assumed cause of stroke between the two groups. Among migraine patients, nine stroke were considered of 
cardio-embolic origin (37.5\%), one carotid dissection (4.1\%), eight of atherosclerosis origin $(33.3 \%)$ and $6(25 \%)$ of undetermined or other origin.

Mean time to CT scan was 125 minutes in migraine patients and 127 minutes in the control group, similar in both groups $(\mathrm{p}=0.97)$.

Regarding the radiological findings, proximal occlusion was found within the first segment of the middle cerebral artery in 5 migraine patients and 9 controls, within the second segment in 5 migraine patients and 14 controls and within the cervical carotid artery in 2 migraine patients and 7 controls. All but one carotid occlusion were associated with occlusion within middle cerebral artery.

We did not find any difference of absolute mismatch value between the two groups $(6,9 \%$ Vs $13 \%, \mathrm{p}=0,46)$. A no-mismatch pattern was equally found in migraine patients and controls $(0 \%$ vs $3.8 \%, p=1)$. The frequency histogram of mismatch values displayed a single cluster of patients in low ratios, with no difference between control and migraine patients (Figure 2). The final infarct volume measured on MRI was not significantly different between migraine and control groups $(30.3 \mathrm{ml} \mathrm{Vs} 51.3 \mathrm{ml}, \mathrm{p}=0.38)$.

These radiological findings remained unchanged when restricting to the 8 patients with aura migraine only: the absolute mismatch value was also $6.9 \%$. No statistical analysis was performed considering the small number of patients.

The rate of reperfusion treatment was $79 \%$ in migraine patients: 19 underwent thrombolysis and three thrombectomy. This rate was equivalent, of $71 \%$, in the control group: 36 patients underwent thrombolysis and 9 thrombectomy. Hemorrhagic transformation occurred in three (14\%) migraine patients and in $15(29 \%)$ control patients, 7 of which were significant (PH1 and $\mathrm{PH} 2$ scores according to the ECASS scale). This rate was similar between the two groups $(\mathrm{p}=0.23)$. On the control MRI, the revascularization rate was $7 / 12(58,3 \%)$ in migraine patients and 27/31 (87\%) in controls with proximal occlusion $(\mathrm{p}=0.09)$. 
Multivariate analysis confirmed the absence of difference between groups in terms of mismatch after adjustment on age, sex and presence of proximal occlusion on initial CT scan $(p=0.22)$. 


\section{DISCUSSION}

In this study in CT perfusion within the 4.5 hours of stroke onset, migraine patients did not display more no-mismatch pattern than controls.

This deviates from the conclusion of previous studies and may be due to our earlier time from stroke onset to CT scan. Indeed, our study showed a similar pattern in CT perfusion between migraine and control patients, possibly representing an early picture of the infarct growth during acute ischemia. In this study, the median time to CT scan was 2 hours, compared to 3 hours in Pezzini's study [5] and 7 hours in Mawet's study [4]. Herein, due to this earlier timing, all patients in both groups were clustered in the low ratio of mismatch value. Previous studies with delayed perfusion CT showed a more flat distribution in both groups with an upsurge in the high ratio for the migraine patients. No details regarding patients who received MRI or CT perfusion within the first 4.5 hours were available in those two studies [4], [5]. Apart from timing, no other relevant difference in our study compared with the previous ones in terms of age, gender, cause of stroke or site of occlusion can explain the diverging results. Similarly to animal studies, this would imply an accelerated growth of infarct and not a constant and steady sensitivity to ischemia [1]. Despite the absence of a prominent nomismatch pattern in the early hours of stroke, migraine may lead to accelerated loss of viable tissue at risk for infarction but measurable from a certain time only. It has been suggested that higher number of cortical spreading depression (CSD) accelerates the growth of the necrosis core and that area of hypoperfusion directly correlates with CSD frequency [11, 12]. Migraine status could influence the rate of progression to infarction through enhanced susceptibility to developing CSD during stroke [13,14]. Indeed, previous study showed even a stronger association between no-mismatch pattern and migraine with aura, in which the role of CSD is clearly established $[4,3,15]$. 
In animal models, accelerated infarct growth would be explained by faster anoxic depolarization and more frequent peri-infarct CSD. Observational studies showed a frequency of zero to ten CSD in an hour [11]. The number of CSD developed in human brain during ischemia remains unknown. Since time is necessary to the propagation of CSD and the accumulation of their negative effects, measurable difference in perfusion $\mathrm{CT}$ in migraine patients may appear after a few hours [16]. Further studies addressed to analyse the necrosis growth over time with repeated perfusion CT or MRI for each patient are needed.

One intriguing point is the similar final infarct volume between migraine patients and controls, in our study as in previous one [5]. This deviates from previous animal studies, where FMH1 mutant mice developed larger infarct after occlusion of the middle cerebral artery [2]. Increased compensatory mechanisms to brain ischemia, possibly through ischemic preconditioning may explain the absence of larger infarct in human migraineurs despite an accelerated growth of necrosis [5]. Ischemic preconditioning involves that cerebral tissue with previous ischemic events shows better tolerance to subsequent events [17]. Indeed, several studies have demonstrated that CSD could induce ischemic preconditioning in animal models, although the exact pathway is still debated $[18,19]$. After experiencing many CSD over the years, migraine patients may partially compensate the growth of infarct, even if accelerated.

Another possible explanation could be that the perfusion abnormalities displayed in migraine patients have been falsely increased by repeated CSD without indicating a perfect match with the growth of necrosis. Moreover, given the retrospective nature of this study, we were not able to accurately record whether a migraine attack had occurred during CT perfusion in our patients. This would probably have also had an impact on CT perfusion abnormalities. Indeed, reversible cerebral blood flow associated with elevated mean transient time has already been reported during migraine aura [20] but this needs to be confirmed by larger studies. 
Some limitations in this study should be considered. The small number of patients and the retrospective character do not allow us to establish definitive conclusions. The small proportion of migraine with aura might also have prevented significant differences to be detected. Nevertheless, reliable assumptions can be made teaming up our findings and previous studies given that the only difference between those studies is the delay from symptoms onset to perfusion imaging. Another important point to discuss is the choice of nomismatch with the cut-off of $83 \%$ as primary endpoint. Given our early timing of CT scans, almost no patient has reached this no-mismatch level. Nevertheless, no difference was found in terms of necrosis and penumbra volumes, nor of their ratio in absolute value. As post-hoc analysis, we used a different ratio, with the cut-off of $38 \%$. This cut-off has been described in literature as providing the highest sensitivity and specificity for identifying patients in whom reperfusion was associated with a favorable response [21]. No migraine patient and only one control showed a ratio necrosis/ ischemia higher than $38 \%$.

We also had a look at the « extremely small ratio » patients, and arbitrarily used the cut-off of $10 \%$ : Seven migraineurs $(33 \%)$ showed a ratio higher than $10 \%$ compared to 13 controls (25\%). It was also similar between groups according to Chi-square test $(\mathrm{p}=0.49)$. This secondary analyses must be interpreted with great caution, but seem to confirm our results with a similar pattern of mismatch between migraine patients and controls during the first few hours of a stroke.

To conclude, a history of migraine may lead to malignant progression of ischemia with penumbra quickly recruited into the infarct core but occurring only after several hours from symptoms onset and not initially. This would make the precocity of reperfusion therapies all the more crucial in migraine patients. Further larger and prospective study to properly address this issue is needed 
ACKNOWLEDGEMENT: We would like to thank the patients and their families for their help with this project.

DISCLOSURE STATEMENT: “The authors have no conflicts of interest to disclose."

\section{AUTHORS CONTRIBUTION:}

IF: collection, analysis and interpretation of data, drafting of manuscript

LG: analysis and interpretation of data, drafting and revision of manuscript.

AC: collection and analysis of data

EH: Statistical analysis and interpretation of data

OO: Recruitment of subjects, drafting of manuscript

AT: Recruitment of subjects, drafting of manuscript

BH: Recruitment of subjects, drafting of manuscript

DM: Drafting and revision of manuscript

EG: Interpretation of data and manuscript revision

EGM: study concept, recruitment of subjects, interpretation of data and manuscript revision.

\section{REFERENCES}

[1] Eikermann-Haerter K, Lee JH, Yalcin N, Yu ES, Daneshmand A, Wei Y, et al. Migraine Prophylaxis, Ischemic Depolarizations, and Stroke Outcomes in Mice. Stroke. 2015 Jan;46(1):229-36.

[2] Eikermann-Haerter K, Lee JH, Yuzawa I, Liu CH, Zhou Z, Shin HK, et al. Migraine mutations increase stroke vulnerability by facilitating ichemic depolarizations. Circulation. 2012 Jan; 17;125(2):335-45. 
[3] Eikermann-Haerter, K \& Ayata, C. Cortical spreading depression and migraine. Curr Neurol Neurosci Rep 10. 2010; 167-173.

[4] Mawet J, Eikermann-Haerter K, Park KY, Helenius J, Daneshmand A, Pearlman L, et al. Sensitivity to acute cerebral ischemic injury in migraineurs: A retrospective case-control study. Neurology. 2015 Dec 1;85(22):1945-9.

[5] Pezzini A, Busto G, Zedde M, Gamba M, Zini A, Poli L, et al. Vulnerability to infarction during cerebral ischemia in migraine sufferers. Stroke. 2018 Mar;49(3):573-8.

[6] Ogata, T, Christensen S, Nagakane Y, Ma H, Campbell BCV, Churilov L,et al. The effects of alteplase 3 to 6 hours after stroke in the EPITHET-DEFUSE combined dataset: post hoc case-control study. Stroke.2003; 44(1), 87-93.

[7] Prabhakaran, S, Ruff I. \& Bernstein RA. A. Acute stroke intervention: a systematic review. JAMA. 2015 Apr; 313 (14), 1451-1462.

[8] Headache Classification Subcommittee of The International Headache Society. The International Classification of Headache Disorders 3rd edition. Cephalalgia 2018 Jan;38(1):1211

[9] Wu O, Østergaard L, Weisskoff RM, Benner T, Rosen BR, Sorensen AG. Tracer arrival timing-insensitive technique for estimating flow in MR perfusion-weighted imaging using singular value decomposition with a block-circulant deconvolution matrix. Magn Reson Med. 2003 Jul;50(1):164-74.

[10] Bivard A, Levi C, Spratt N, Parsons M. Perfusion CT in acute stroke: a comprehensive analysis of infarct and penumbra. Radiology. 2013 May;267(2):543-50.

[11] Nakamura H, Strong AJ, Dohmen C, Sakowitz OW, Vollmar S, Sué M, et al. Spreading depolarizations cycle around and enlarge focal ischaemic brain lesions. Brain. 2010 Jul;133(7):1994-2006. 
[12] Eikermann-Haerter K, Dileköz E, Kudo C, Savitz SI, Waeber C, Baum MJ, et al. Genetic and hormonal factors modulate spreading depression and transient hemiparesis in mouse models of familial hemiplegic migraine type 1. Journal of Clinical Investigation. 2009 Jan; 119(1):99- 109.

[13] Eikermann-Haerter K. Spreading depolarization may link migraine and stroke. Headache. $2014 \mathrm{Jul} ; 54(7): 1146-57$.

[14] Lauritzen M. Cortical spreading depression in migraine. Cephalalgia. 2001 Sep;21(7):757-60.

[15] Leão AAP. Spreading depression of activity in the cerebral cortex. J Neurophysiol 1944 Nov ;7(6): 359-390.

|16] Hartings JA, Rolli ML, Lu XC, Tortella FC. Delayed secondary phase of peri-infarct depolarizations after focal cerebral ischemia: relation to infarct growth and neuroprotection. $\mathbf{J}$ Neurosci. 2003 Dec; 23(37):11602-10.

[17] Gidday JM. Cerebral preconditioning and ischaemic tolerance. Nat Rev Neurosci. 2006 Jun;7(6):437-48.

[18] Shen P, Hou S, Zhu M, Zhao M, Ouyang Y, Feng J. Cortical spreading depression preconditioning mediates neuroprotection against ischemic stroke by inducing AMP-activated protein kinase-dependent autophagy in a rat cerebral ischemic/reperfusion injury model. $\mathbf{J}$ Neurochem. 2017 Mar;140(5):799-813.

[19] Viggiano E, Monda V, Messina A, Moscatelli F, Valenzano A, Tafuri D, et al. Cortical spreading depression produces a neuroprotective effect activating mitochondrial uncoupling protein-5. Neuropsychiatr Dis Treat. 2016 Jul;12:1705-10.

[20] Shah L, Rana S, Valeriano J, Scott TF. Reversible CT perfusion abnormalities in patient with migraine variant: a two phase process. Clin Neurol Neurosurg. 2013 Jun;115(6):830-2. 
[21] Kakuda W, Lansberg MG, Thijs VN, Kemp SM, Bammer R, Weschler LR et al. Optimal definition of PWI/DWI mismatch in acute ischemic sytroke patients. J Cerebral Blood Flow Metab. 2008;28(5): 887-891. 
TABLES AND FIGURE CAPTIONS

Table 1: Demographic and clinical characteristics according to migraine status

\begin{tabular}{|c|c|c|c|}
\hline & $\begin{array}{l}\text { Migraine } \\
\text { patients }(n=24)\end{array}$ & Controls $(\mathrm{n}=51)$ & $p$ \\
\hline Migraine with aura, n (\%) & $8(33 \%)$ & NA & NA \\
\hline Age, y, mean +/- SD & $63+/-13.7$ & $68.7+/-12.4$ & 0,09 \\
\hline Female, n (\%) & $14(58 \%)$ & $17(33 \%)$ & 0,04 \\
\hline \multicolumn{4}{|l|}{ Risk factors, $n(\%)$} \\
\hline High blood pressure & $13(54 \%)$ & $27(53 \%)$ & 1 \\
\hline Diabetes mellitus & $3(13 \%)$ & $6(12 \%)$ & 1 \\
\hline Smoking history & $8(33 \%)$ & $24(47 \%)$ & 0,32 \\
\hline Hyperlipidemia & $9(38 \%)$ & $20(39 \%)$ & 1 \\
\hline Personnal vascular history & $3(13 \%)$ & $15(30 \%)$ & 0,15 \\
\hline Familial vascular history & $2(8 \%)$ & $6(12 \%)$ & 1 \\
\hline \multicolumn{4}{|l|}{ Clinical scores, median +/- SD } \\
\hline RANKIN at admission & $0+/-0,3$ & $0+/-0,3$ & 0,85 \\
\hline NIHSS at admission & $5,5+/-6,9$ & $10,0+/-7,5$ & 0,26 \\
\hline RANKIN at discharge & $0+/-1,5$ & $1,0+/-1,6$ & 0,23 \\
\hline NIHSS at discharge & $0+/-3,6$ & $1,0+/-5,3$ & 0,1 \\
\hline Stroke etiologic subtype & $* * *$ & $* * *$ & 0,98 \\
\hline Intravenous thrombolysis ,\% (n) & $19(79 \%)$ & $36(71 \%)$ & 0,58 \\
\hline Mechanical thrombectomy, \% (n) & $3(13 \%)$ & $9(18 \%)$ & 0,74 \\
\hline
\end{tabular}

(*) significant. (**) Non applicable. (***) Categories non detailed, SD Standard deviation 
Table 2: Radiological characteristics according to migraine status

\begin{tabular}{|c|c|c|c|}
\hline & $\begin{array}{l}\text { Migraine } \\
\text { patients }(n=24)\end{array}$ & Controls $(n=51)$ & $p$ \\
\hline \multicolumn{4}{|l|}{ Time from onset in minutes, mean +/- SD } \\
\hline To CT scan & $125+/-55$ & $127+/-54$ & 0,97 \\
\hline To intravenous thrombolysis & $173+/-51$ & $154+/-50$ & 0,22 \\
\hline To mechanical thrombectomy & $235+/-18$ & $249+/-76$ & 0,61 \\
\hline Occlusion on initial angiogram, n (\%) & $12(50 \%)$ & $31(60.7 \%)$ & \\
\hline Within $1^{\text {st }}$ segment of middle cerebral artery & $5(20.8 \%)$ & $9(17.6 \%)$ & 0,37 \\
\hline Within 2nd segment of middle cerebral artery & $5(20.8 \%)$ & $14(27.4 \%)$ & \\
\hline Within cervical carotid artery & $2(8 \%)$ & $7(13.7 \%)$ & \\
\hline Within anterior cerebral artery & 0 & 0 & \\
\hline \multicolumn{4}{|l|}{ Volumes, ml, mean +/- SD } \\
\hline Necrosis volume on perfusion CT & $8,6+/-18,5$ & $8,8+/-16,2$ & 0,66 \\
\hline Ischemia volume on perfusion $\mathrm{CT}$ & $69,4+/-65,0$ & $71,1+/-68,9$ & 0,95 \\
\hline Stroke volume on MRI & $30,3+/-46,8$ & $52,3+/-70,6$ & 0,31 \\
\hline Necrosis volume/Ischemia volume, mean & $6,9 \%$ & $13,1 \%$ & 0,48 \\
\hline No mismatch pattern, $\%$ & 0 & $2(3,9 \%)$ & 1 \\
\hline
\end{tabular}

\section{Figure 1: Flow chart}

Figure 2: Frequency histogram of mismatch values for migraine patients and control group 


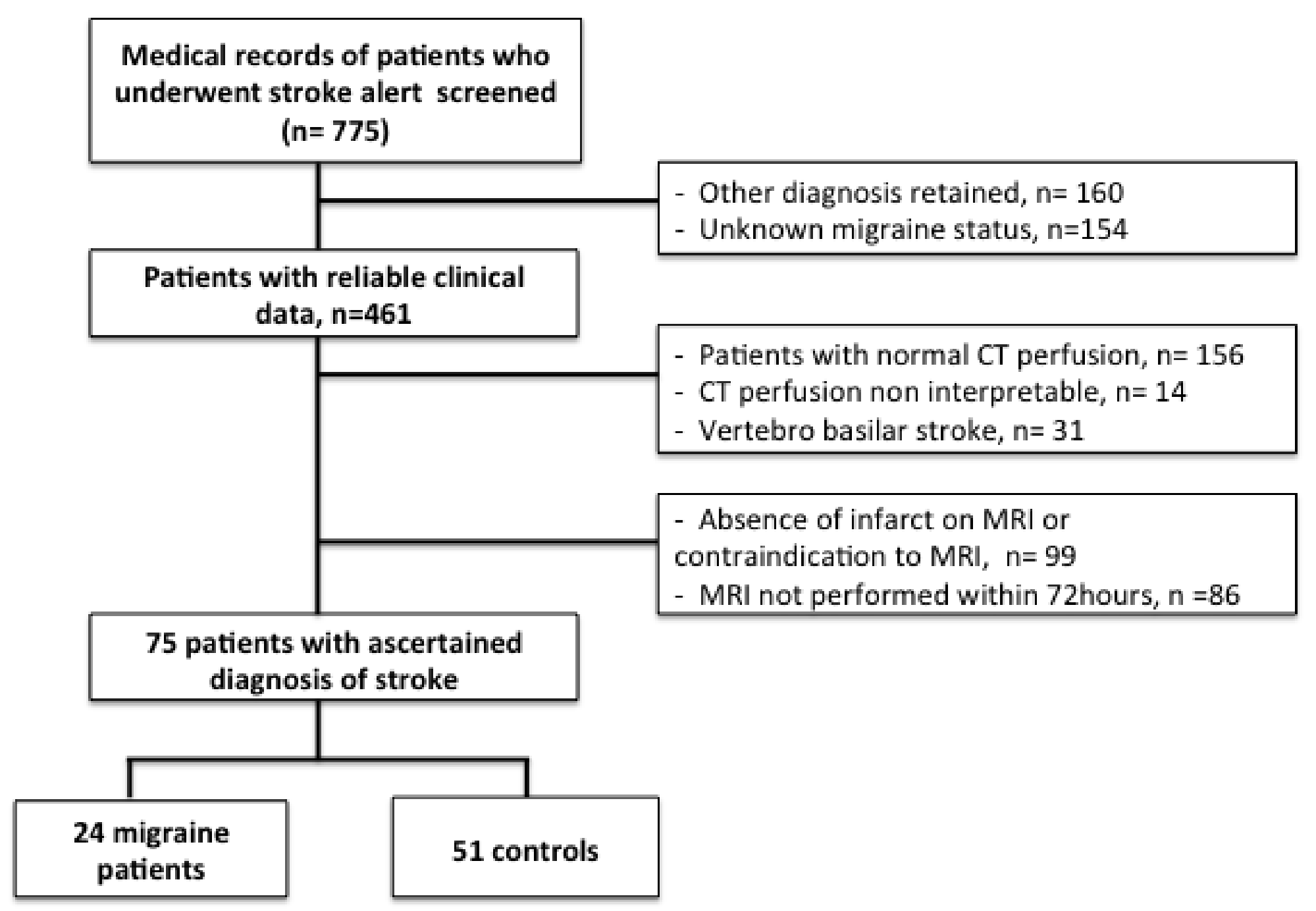


Percentage of population

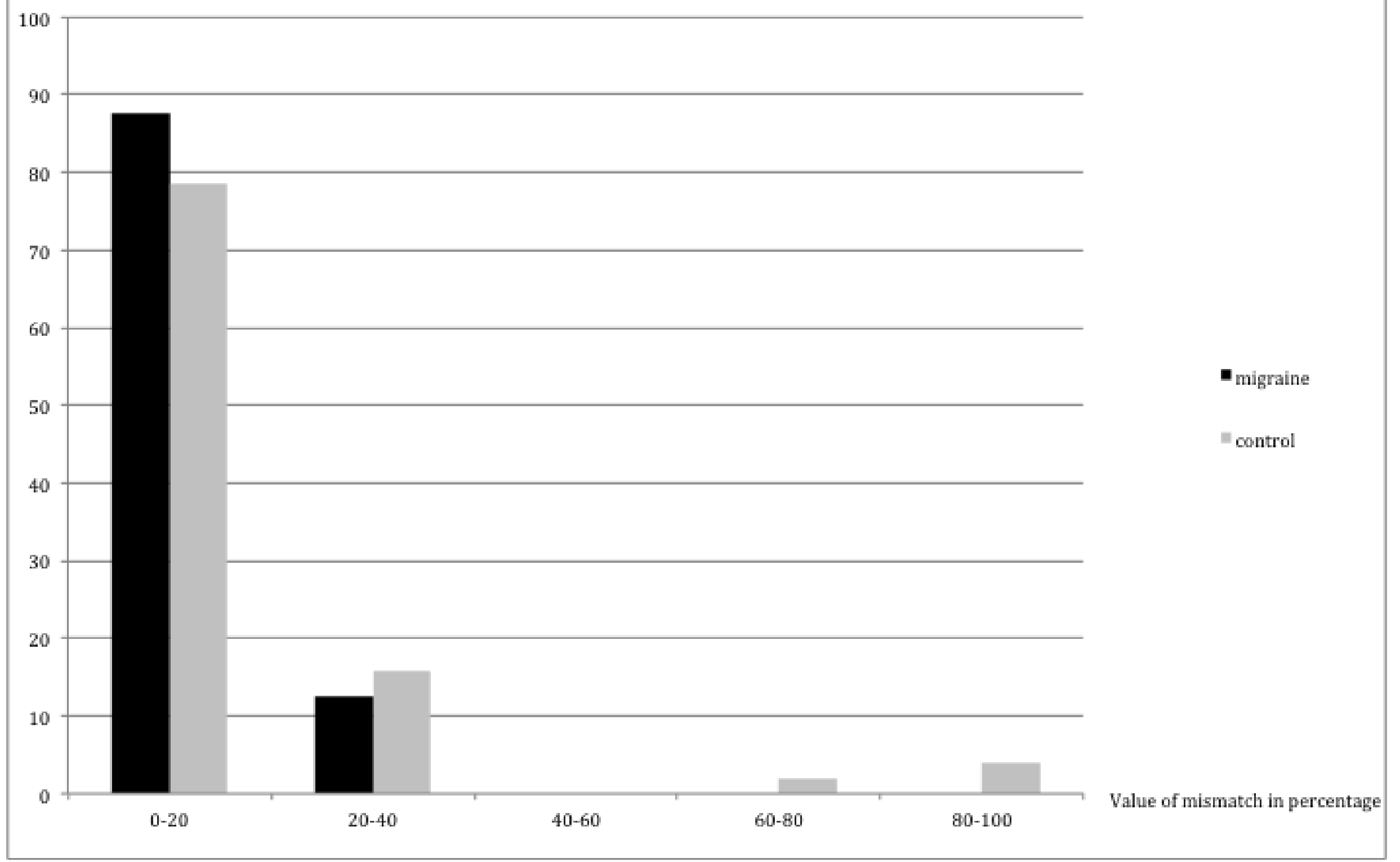

